\title{
OPEN Safety and efficacy of Melissa officinalis extract containing rosmarinic acid in the prevention of Alzheimer's disease progression
}

\author{
Moeko Noguchi-Shinohara ${ }^{1,2}$, Kenjiro Ono ${ }^{1,3}$, Tsuyoshi Hamaguchi ${ }^{1}$, Toshitada Nagai ${ }^{4}$, \\ Shoko Kobayashi ${ }^{5}$, Junji Komatsu ${ }^{1,2}$, Miharu Samuraki-Yokohama ${ }^{1}$, Kazuo Iwasa ${ }^{1,6}$, \\ Kunihiko Yokoyama ${ }^{7}$, Hiroyuki Nakamura ${ }^{8}$ \& Masahito Yamada ${ }^{1 凶}$
}

We conducted a randomized placebo-controlled double-blind 24-week trial using Melissa officinalis (M. officinalis) extract richly containing rosmarinic acid (RA) on patients with mild dementia due to Alzheimer's disease (AD) with the aim to examine the safety and tolerability (primary endpoint) of RA ( $500 \mathrm{mg}$ daily) and its clinical effects and disease-related biomarker changes (secondary endpoints). Patients ( $n=23$ ) diagnosed with mild dementia due to probable AD were randomized to either the placebo or $M$. officinalis extract group. No differences in vital signs or physical and neurologic examination results were detected between the $M$. officinalis and placebo groups. No serious adverse events occurred. There were no significant differences in cognitive measures; however, the mean Neuropsychiatric Inventory Questionnaire (NPI-Q) score improved by 0.5 points in the $M$. officinalis group and worsened by 0.7 points in the placebo group between the baseline and 24-week visit, indicating a significant difference $(P=0.012)$. No significant differences were apparent in diseaserelated biomarkers between the groups. $M$. officinalis extract containing $500 \mathrm{mg}$ of RA taken daily was safe and well-tolerated by patients with mild dementia due to $A D$. Our results suggest that RA may help prevent the worsening of AD-related neuropsychiatric symptoms. Trial registration: The registration number for this clinical trial is UMIN000007734 (16/04/2012).

Alzheimer's disease (AD) is characterized by parenchymal and vascular amyloid deposits of amyloid $\beta$-protein $(\mathrm{A} \beta)$ and neurofibrillary tangles formed by the microtubule-associated protein tau ${ }^{1}$. Because the deposition of $\mathrm{A} \beta$ aggregates in the brain occurs approximately 25 years before the appearance of clinical symptoms, it has been proposed that treatments targeting $A \beta$ aggregation, including formation of neurotoxic $A \beta$ oligomers, should be implemented early in the disease process ${ }^{2}$. Dietary modifications and/or nutraceutical supplementation are appropriate methods for the prevention or treatment of $\mathrm{AD}$.

Rosmarinic acid (RA) is an ester of caffeic acid and 3,4-dihydroxiphenyllactic acid. It has several interesting biological properties, including antioxidant, anti-inflammatory, antimutagenic, anti-bacterial, and antiviral properties $^{3}$. We previously reported that RA inhibits the formation of $A \beta$ fibrils, comprising $A \beta_{1-40}$ and $A \beta_{1-42}$, and destabilizes preformed $A \beta$ fibrils in vitro in a dose-dependent manner ${ }^{4}$. RA also inhibits the oligomerization of both $A \beta_{1-40}$ and $A \beta_{1-42}$ in vitro ${ }^{5}$. Moreover, our long-term potentiation and depression assays using hippocampal slices indicated that RA decreases $A \beta$ oligomer-induced synaptic toxicities ${ }^{5}$. Long-term potentiation and depression are considered important neurophysiological models of memory and learning and are used as

\footnotetext{
${ }^{1}$ Department of Neurology and Neurobiology of Aging, Kanazawa University Graduate School of Medical Sciences, Kanazawa University, 13-1 Takara-machi, Kanazawa 920-8640, Japan. ${ }^{2}$ Department of Preemptive Medicine for Dementia, Kanazawa University Graduate School of Medical Sciences, Kanazawa University, Kanazawa, Japan. ${ }^{3}$ Division of Neurology, Department of Internal Medicine, Showa University School of Medicine, Hatano-dai, Shinagawa-ku, Tokyo 142-8666, Japan. ${ }^{4}$ Department of Food and Life-Science, Takasaki University of Health and Welfare, Gunma, Japan. ${ }^{5}$ Research Center for Food Safety, Graduate School of Agricultural and Life Sciences, The University of Tokyo, Tokyo, Japan. ${ }^{6}$ Department of Health and Medical Sciences, Ishikawa Prefectural Nursing University, Kahoku, Japan. ${ }^{7}$ Department of Thyroidology, Public Central Hospital of Matto Ishikawa, Hakusan, Japan. ${ }^{8}$ Department of Environmental and Preventive Medicine, Kanazawa University Graduate School of Medical Sciences, Kanazawa University, Kanazawa, Japan. ${ }^{\circledR}$ email: m-yamada@med.kanazawa-u.ac.jp
} 


\begin{tabular}{|l|l|l|l|}
\hline & $\begin{array}{l}\text { Melissa officinalis } \\
\text { extract group }(\mathbf{n}=\mathbf{1 2})\end{array}$ & $\begin{array}{l}\text { Placebo } \\
\text { group }(\mathbf{n}=\mathbf{1 1})\end{array}$ & P value \\
\hline Age (years) & $73.42 \pm 5.00$ & $72.45 \pm 7.53$ & 0.786 \\
\hline No. of women, $\mathrm{n}(\%)$ & $5(41.6)$ & $6(54.5)$ & 0.684 \\
\hline Years of education & $11.92 \pm 1.00$ & $12.36 \pm 2.20$ & 1.000 \\
\hline APOE E4 carrier $(\%)$ & 83.3 & 63.63 & 0.371 \\
\hline MMSE score & $22.92 \pm 1.83$ & $23.55 \pm 1.81$ & 0.449 \\
\hline ADAS-cog score & $25.92 \pm 6.46$ & $24.30 \pm 4.37$ & 0.456 \\
\hline DAD score & $28.00 \pm 8.83$ & $26.27 \pm 7.52$ & 0.487 \\
\hline NPI-Q score & $4.58 \pm 3.63$ & $4.82 \pm 1.72$ & 0.740 \\
\hline CDR score $=1(\%)$ & 100 & 100 & 1.000 \\
\hline${ }^{11}$ C-PiB PET SUVR value & $1.81 \pm 0.21$ & $1.81 \pm 0.21$ & 1.000 \\
\hline${ }^{18}$ F-FDG PET Z-score & $2.07 \pm 0.71$ & $2.14 \pm 0.82$ & 0.833 \\
\hline Head MRI VSRAD value & $3.87 \pm 1.34$ & $3.82 \pm 1.21$ & 0.976 \\
\hline CSF-A $\beta_{1-42}$ concentration $(\mathrm{pg} / \mathrm{mL})$ & $458.82 \pm 140.80$ & $447.78 \pm 112.54$ & 1.000 \\
\hline CSF-tau concentration $(\mathrm{pg} / \mathrm{mL})$ & $828.80 \pm 442.61$ & $806.25 \pm 516.82$ & 0.829 \\
\hline CSF-ptau concentration $(\mathrm{pg} / \mathrm{mL})$ & $95.09 \pm 40.57$ & $78.22 \pm 32.06$ & 0.295 \\
\hline
\end{tabular}

Table 1. Demographics and baseline clinical characteristics. Data represent mean \pm standard deviation (SD) or percent values. Significant differences between groups were determined by analysis of variance (ANOVA) and Chi-square $\left(\chi^{2}\right)$ test, where appropriate. $A \beta$ amyloid $\beta$-protein; $A D A S$ - $\operatorname{cog}$ cognitive subscale of the Alzheimer's Disease Assessment Scale; $A P O E$ apolipoprotein E; CDR clinical dementia rating; CSF cerebrospinal fluid; $D A D$ Disability Assessment for Dementia; FDG fluorodeoxyglucose; MMSE Mini-Mental State Examination; NPI-Q Neuropsychiatric Inventory-Questionnaire; PET positron emission tomography; ptau phosphorylated tau 181p. PiB Pittsburgh compound-B; SUVR standardized uptake value ratio; tau, total tau protein; VSRAD Voxel-Based Specific Regional Analysis System for Alzheimer's disease.

experimental models of neuronal plasticity ${ }^{6}$. In intracerebroventricular $A \beta_{25-35}$ injection mice model studies, intracerebroventricular injection of RA prevents memory impairment and A $\beta$-induced neurotoxicity by scavenging $\mathrm{ONOO}^{-7}$. We systematically investigated the effects of phenolic compounds on AD transgenic mice (Tg2576) model ${ }^{8}$. Mice were fed five phenolic compounds (curcumin, ferulic acid, myricetin, nordihydroguaiaretic acid, and RA) ( $1 \mathrm{~g} / \mathrm{kg} /$ day) for 10 months from the age of 5 months ${ }^{8}$. Of the compounds tested, RA appeared to be the most attractive molecule for preventing $\mathrm{AD}$, because it inhibited both the oligomerization and deposition of $A \beta^{8}$. Moreover, we recently demonstrated that monoamine suppresses $A \beta$ aggregation ${ }^{9}$, suggesting that an RA-triggered increase in monoamine secretion in the brain is beneficial for the treatment of AD.

RA is present in various herbs such as perilla (Perilla frutescens L. $)^{10}$, rosemary (Rosmarinus officinalis L. $)^{11}$, sage (Salvia officinalis L. $)^{12}$, and lemon balm [Melissa officinalis (M. officinalis) L. $]^{13}$. Extracts of M. officinalis are reported to have positive effects on cognitive performance in healthy participants ${ }^{14,15}$. In a 16-week, randomized, double-blind, placebo-controlled study, patients with mild to moderate AD treated with M. officinalis extract showed significantly higher cognitive function, with no side effects, compared with patients administered placebo ${ }^{16}$; however, the amount of RA contained in the M. officinalis extract was not analyzed, and no disease-related biomarkers were evaluated ${ }^{16}$. Previously, we prepared M. officinalis extract richly containing RA and showed that a single dose of M. officinalis extract containing $500 \mathrm{mg}$ of RA is safe and tolerable in healthy individuals ${ }^{17}$.

Here, we performed a randomized double-blind placebo-controlled 24-week study of M. officinalis extract containing RA in patients with mild dementia due to $\mathrm{AD}$, with an open-label extension to 48 weeks. The primary endpoint was the safety and tolerability of the M. officinalis extract, and the secondary endpoints the clinical efficacy of M. officinalis extract and changes in AD biomarkers.

\section{Results}

Primary outcome. A total of 23 patients were randomized to either the M. officinalis group (12 patients) or the placebo group (11 patients). Table 1 shows the demographic and clinical characteristics of patients included in the full-analysis set.

Three patients withdrew from the study between the baseline and 24-week follow-up visit; two patients (one from the M. officinalis group and one from the placebo group) withdrew because they had to be admitted into a nursing home, and another patient from the M. officinalis group withdrew because caregivers lost interest in the trial. A total of 20 patients (83.3-90.9\% of the patients in each group) completed part 1 of the trial (Fig. 1 and Table 1), and all these patients entered part 2 (extension period).

No differences were detected in the vital signs and physical and neurological examinations between the $M$. officinalis and placebo groups. Overall, adverse events occurred in $41.6 \%$ of the patients in the M. officinalis group and $45.5 \%$ of the patients in the placebo group $(P=1.000)$. Regarding concomitant medications, no apparent adverse events were reported. None of these adverse events were serious or led to the discontinuation of the trial regimen (Table 2). 


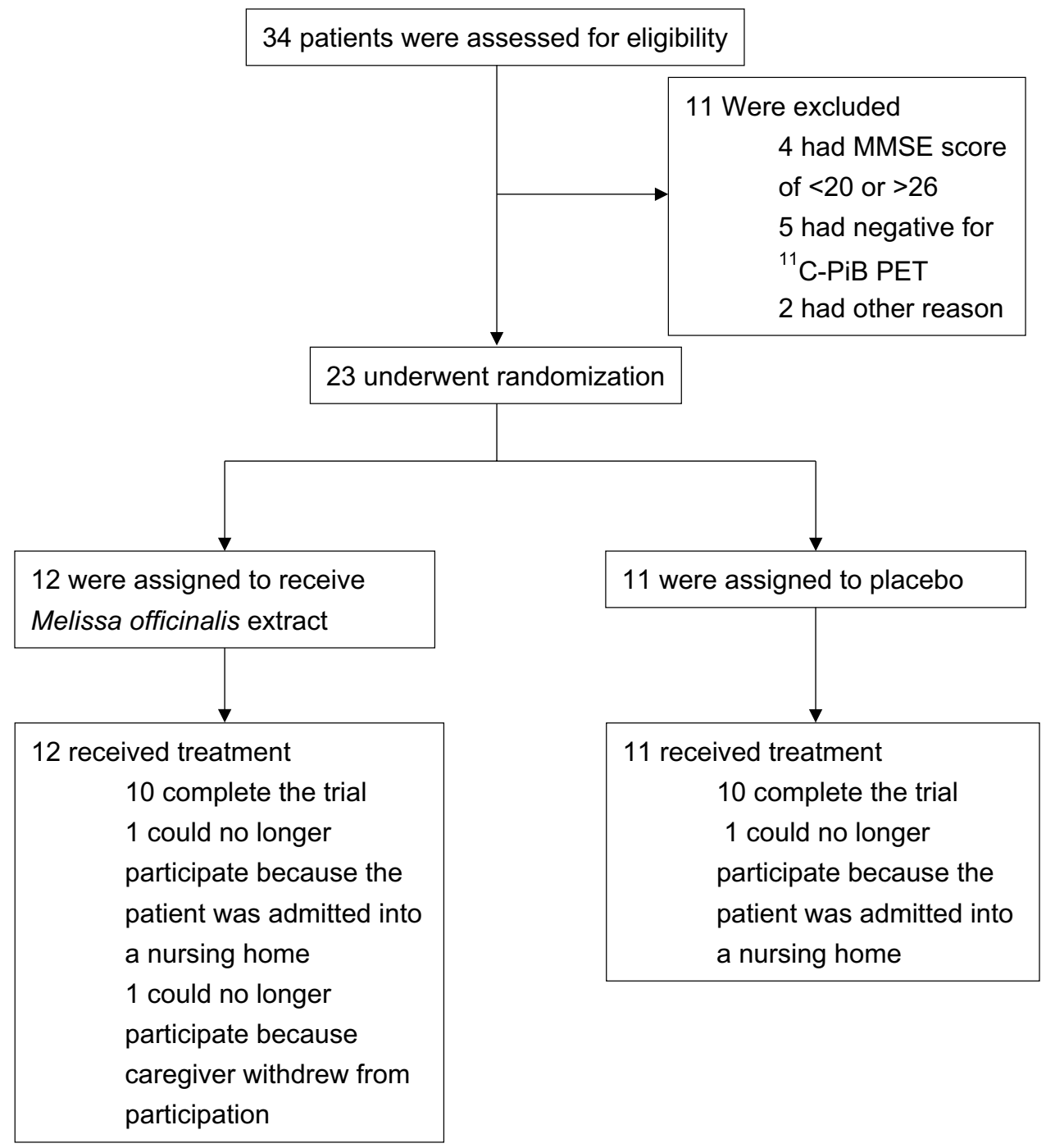

Figure 1. Randomization, trial-group assignment, and follow-up of the trial. Scores of the Mini-Mental State Examination (MMSE) range from 0 to 30, with lower scores indicating poorer cognitive performance. In the positron emission tomography (PET) study, the mean cortical standardized uptake value ratio (SUVR) was calculated using the ratio of the SUV between the whole cerebrum region of interest (ROI) and the reference cerebellum ROI. ${ }^{11} \mathrm{C}$-Pittsburgh compound-B (PiB) PET negative: mean cortical ${ }^{11} \mathrm{C}-\mathrm{PiB}$ SUVR values less than 1.45 indicated a negative result for Alzheimer's disease (AD) pathology. MMSE Mini-Mental State Examination; $P E T$ positron emission tomography; $P i B$ Pittsburgh compound-B; ROI region of interest; SUVR standardized uptake value ratio.

Serum levels of liver function indicators [aspartate aminotransferase (AST), alanine aminotransferase (ALT), lactate dehydrogenase (LDH), and total bilirubin (T-bil)] and kidney function indicators [blood urea nitrogen (BUN) and creatinine (Cr)] and hematology findings showed no significant differences between the M. officinalis and placebo groups. Adherence to treatment of the M. officinalis and placebo groups were found to be $77.42 \%$ and $87.45 \%$, respectively, in the full-analysis set.

Secondary outcomes. The score changes from baseline to the 8-, 16-, and 24-week follow-up visits showed no significant differences in Mini-Mental State Examination (MMSE) cognitive subscale of the Alzheimer's Assessment Scale (ADAS-cog), Disability Assessment for Dementia scale (DAD) clinical dementia rating (CDR) between the treatment groups (Table 3).

Assessment of the neuropsychiatric effect of $M$. officinalis extract on the Neuropsychiatric Inventory-Questionnaire (NPI-Q) score from baseline to the 8-, 16-, and 24-week timepoints and baseline to the 8-, 16-, 24-, 32-, 40 -, and 48-week timepoints using per protocol set revealed the time $\times$ treatment interaction effect as significant $(F=4.028, P=0.012$ and $F=5.766, P<0.001$, respectively; Fig. 2 and Table 3$)$. Using full-analysis set, time $\times$ treatment interaction effects were significant for the NPI-Q score from baseline to 24-week follow-up and 48-week 


\begin{tabular}{|l|l|l|}
\hline Adverse events & $\begin{array}{l}\text { Melissa officinalis } \\
\text { extract group }(\boldsymbol{n}=\mathbf{1 2})\end{array}$ & Placebo group $(\boldsymbol{n}=\mathbf{1 1})$ \\
\hline Bruising & 1 & 0 \\
\hline Headache & 1 & 0 \\
\hline Hematuria & 1 & 0 \\
\hline Upper respiratory infection & 1 & 2 \\
\hline ALT elevation & 0 & $1^{*}$ \\
\hline AST elevation & 0 & $1^{*}$ \\
\hline Hyperkalemia & 0 & 1 \\
\hline Hypokalemia & 0 & 1 \\
\hline Rash maculopapular & 0 & $2\left(1^{\star}\right)$ \\
\hline Weight loss & 0 & 1 \\
\hline
\end{tabular}

Table 2. Number of patients with adverse events. ALT alanine aminotransferase; AST aspartate aminotransferase. ${ }^{\star}$ Adverse events were reported during the extension period.

\begin{tabular}{|c|c|c|c|c|c|c|c|c|}
\hline & & Baseline & 24 weeks & 48 weeks & $\begin{array}{l}\text { Mean change } \\
\Delta \text { Baseline- } 24 \text { weeks }\end{array}$ & $P$ value & $\begin{array}{l}\text { Mean change } \\
\Delta \text { Baseline-48 weeks }\end{array}$ & $P$ value \\
\hline $\begin{array}{l}\text { MMSE } \\
\text { score }\end{array}$ & $\begin{array}{l}\text { M. officinalis } \\
(n=10) \\
\text { Placebo } \\
\text { group } \\
(n=10)\end{array}$ & $\begin{array}{l}23.40 \pm 1.58 \\
23.70 \pm 1.83\end{array}$ & $\begin{array}{l}22.10 \pm 2.73 \\
22.40 \pm 3.47\end{array}$ & $\begin{array}{l}21.30 \pm 3.86 \\
23.30 \pm 3.78\end{array}$ & $\begin{array}{l}-1.30 \pm 2.36 \\
-1.30 \pm 2.36\end{array}$ & 0.513 & $\begin{array}{l}-2.10 \pm 3.73 \\
-0.40 \pm 2.27\end{array}$ & 0.459 \\
\hline $\begin{array}{l}\text { ADAS-cog } \\
\text { score }\end{array}$ & $\begin{array}{l}\text { M. officinalis } \\
(n=10) \\
\text { Placebo } \\
\text { group } \\
(n=10)\end{array}$ & $\begin{array}{l}23.90 \pm 4.79 \\
24.30 \pm 4.37\end{array}$ & $\begin{array}{l}23.60 \pm 4.84 \\
26.10 \pm 6.17\end{array}$ & $\begin{array}{l}26.30 \pm 3.13 \\
25.90 \pm 8.63\end{array}$ & $\begin{array}{l}-0.30 \pm 4.35 \\
1.80 \pm 3.88\end{array}$ & 0.239 & $\begin{array}{l}2.40 \pm 5.50 \\
1.60 \pm 5.32\end{array}$ & 0.657 \\
\hline DAD score & $\begin{array}{l}\text { M. officinalis } \\
(n=10) \\
\text { Placebo } \\
\text { group } \\
(n=10)\end{array}$ & $\begin{array}{l}30.10 \pm 7.31 \\
27.90 \pm 5.53\end{array}$ & $\begin{array}{l}30.30 \pm 5.74 \\
27.40 \pm 5.91\end{array}$ & $\begin{array}{l}28.60 \pm 5.73 \\
25.20 \pm 8.47\end{array}$ & $\begin{array}{l}0.20 \pm 1.99 \\
-0.50 \pm 3.03\end{array}$ & 0.464 & $\begin{array}{l}-1.50 \pm 4.50 \\
-2.70 \pm 3.77\end{array}$ & 0.828 \\
\hline $\begin{array}{l}\text { NPI-Q } \\
\text { score }\end{array}$ & $\begin{array}{l}\text { M. officinalis } \\
(n=10) \\
\text { Placebo } \\
\text { group } \\
(n=10)\end{array}$ & $\begin{array}{l}4.60 \pm 3.84 \\
4.80 \pm 1.81\end{array}$ & $\begin{array}{l}4.10 \pm 3.51 \\
5.50 \pm 2.17\end{array}$ & $\begin{array}{l}3.50 \pm 3.03 \\
6.10 \pm 2.08\end{array}$ & $\begin{array}{l}-0.50 \pm 1.43 \\
0.70 \pm 1.89\end{array}$ & 0.012 & $\begin{array}{l}-1.10 \pm 2.54 \\
1.30 \pm 2.54\end{array}$ & $<0.001$ \\
\hline CDR score & $\begin{array}{l}\text { M. officinalis } \\
(n=10) \\
\text { Placebo } \\
\text { group } \\
(n=10)\end{array}$ & $\begin{array}{l}1.0(n=10) \\
1.0(n=10)\end{array}$ & $\begin{array}{l}1.0(n=10) \\
1.0(n=9) \\
2.0(n=1)\end{array}$ & $\begin{array}{l}1.0(n=10) \\
1.0(n=9) \\
2.0(n=1)\end{array}$ & - & - & - & - \\
\hline
\end{tabular}

Table 3. Mean and estimated mean changes in clinical assessments from the baseline to 24-week follow-up and the baseline to 48-week follow-up. ADAS-cog cognitive subscale of the Alzheimer's Disease Assessment Scale; $C D R$ clinical dementia rating; DAD Disability Assessment for Dementia; M. officinalis, Melissa officinalis; MMSE Mini-Mental State Examination; NPI-Q Neuropsychiatric Inventory-Questionnaire.

follow-up $(F=4.576, P=0.006$ and $F=5.984, P<0.001)$, respectively. The data of each subject of the NPI-Q scores are summarized in the Supplemental Table.

The effect of time $\times$ treatment interaction on for NPI-Q scores was significant $(P<0.05)$. Open triangles represent the $M$. officinalis group, and open circles represent the placebo group.

The time variable was not significant $(F=0.484, P=0.695)$. The mean NPI-Q scores improved by 0.5 points in the $M$. officinalis group and worsened by 0.7 points in the placebo group between baseline and the 24 -week visit. On further analyzing each NPI-Q subscale, time $\times$ treatment variable of "Irritability/Lability" was significant $(F=4.539, P=0.006)$; the mean scores of "Irritability/Lability" improved by 0.32 points in the M. officinalis group and worsened by 0.23 points in the placebo group between baseline and the 24 -week visit. Regarding the extension period, in which all patients were assigned to the $M$. officinalis extract group, the mean NPI-Q scores worsened by 0.6 points in the placebo group between the 24 -week visit and the 48 -week visit.

Changes in disease-related biomarkers, including ${ }^{11} \mathrm{C}$-Pittsburgh compound-B (PiB) positoron emission tomography (PET) standardized uptake value ratio (SUVR), ${ }^{18} \mathrm{~F}$-fluorodeoxyglucose (FDG) PET z-scores, MRI z-scores, and cerebrospinal fluid (CSF)-A $\beta_{1-42}$, CSF-total tau protein (tau), and CSF-phosphorylated tau $181 \mathrm{p}$ (ptau) concentrations, showed no significant differences between the two treatment groups (Table 4).

Exploratory examination. The mean concentrations of total RA (intact and conjugated forms) in serum samples of the M. officinalis group at the 8-, 16-, and 24-week visits were $52.60 \pm 41.38,48.49 \pm 27.83$, and 


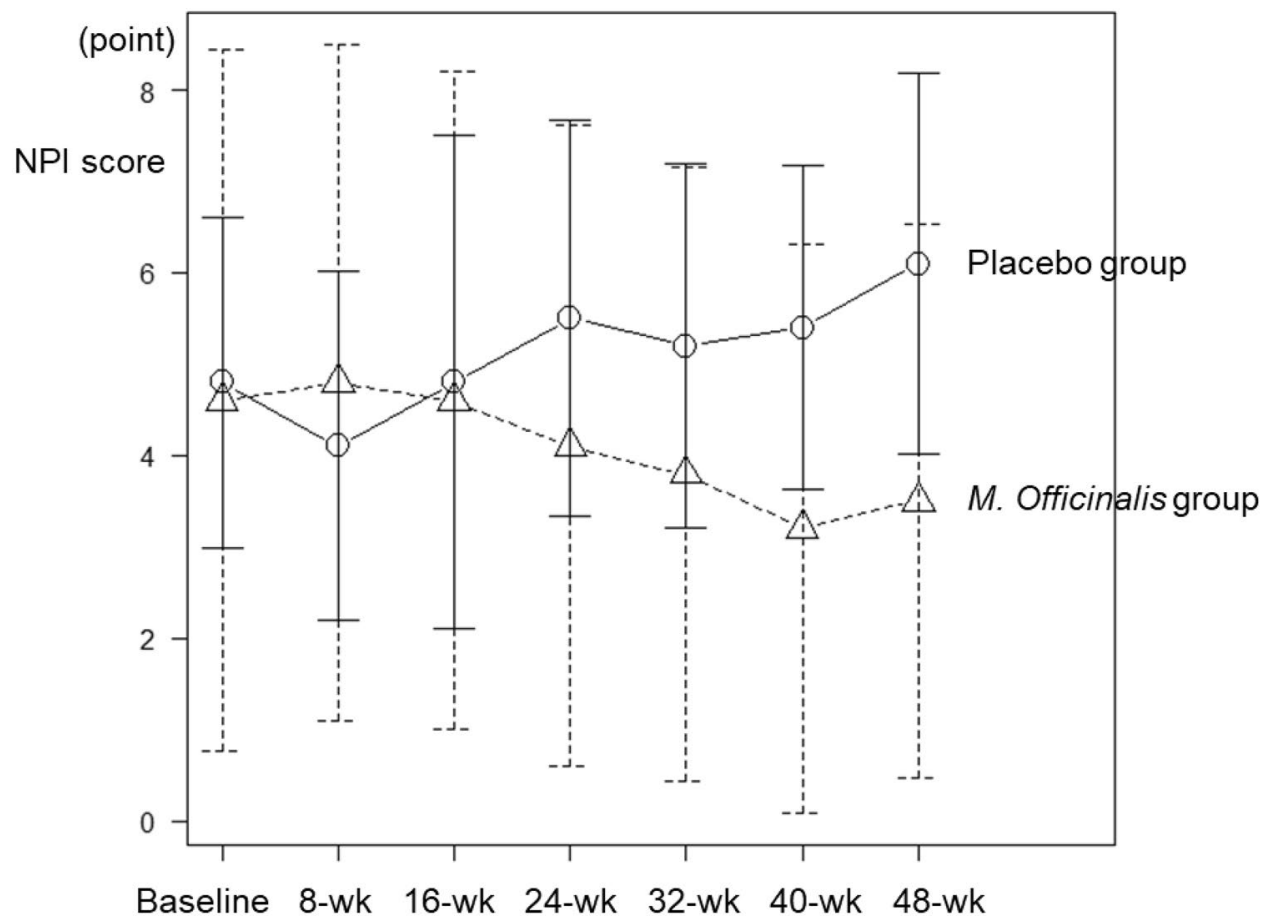

Figure 2. Means of Neuropsychiatric Inventory (NPI-Q) scores at each study time point.

\begin{tabular}{|l|l|l|l|l|l|}
\hline & & Baseline & $\mathbf{2 4}$ weeks & Mean change & P value \\
\hline${ }^{11}$ C-PiB PET SUVR value & $\begin{array}{l}\text { M. officinalis }(n=10) \\
\text { Placebo }(n=9)\end{array}$ & $\begin{array}{l}1.81 \pm 0.22 \\
1.80 \pm 0.19\end{array}$ & $\begin{array}{l}1.76 \pm 0.26 \\
1.79 \pm 0.35\end{array}$ & $\begin{array}{l}-0.05 \pm 0.10 \\
-0.02 \pm 0.30\end{array}$ & 0.838 \\
\hline${ }^{18}$ F-FDG PET Z score & $\begin{array}{l}\text { M. officinalis }(n=10) \\
\text { Placebo }(n=10)\end{array}$ & $\begin{array}{l}1.91 \pm 0.65 \\
2.00 \pm 0.70\end{array}$ & $\begin{array}{l}2.01 \pm 0.75 \\
2.26 \pm 0.97\end{array}$ & $\begin{array}{l}0.10 \pm 0.19 \\
0.26 \pm 0.51\end{array}$ & 0.352 \\
\hline \multirow{2}{*}{ Head MRI VSRAD value } & $\begin{array}{l}\text { M. officinalis }(n=10) \\
\text { Placebo }(n=10)\end{array}$ & $\begin{array}{l}3.59 \pm 1.29 \\
4.02 \pm 1.06\end{array}$ & $\begin{array}{l}3.70 \pm 1.53 \\
4.35 \pm 1.22\end{array}$ & $\begin{array}{l}0.12 \pm 0.41 \\
0.33 \pm 0.47\end{array}$ & 0.286 \\
\hline CSF-A $\beta_{1-42}$ concentration & $\begin{array}{l}\text { M. officinalis }(n=7) \\
\text { Placebo }(n=6)\end{array}$ & $\begin{array}{l}507.86 \pm 142.09 \\
492.17 \pm 101.64\end{array}$ & $\begin{array}{l}490.57 \pm 52.73 \\
435.50 \pm 60.98\end{array}$ & $\begin{array}{l}-17.29 \pm 124.75 \\
-56.67 \pm 75.39\end{array}$ & 0.559 \\
\hline CSF-tau concentration & $\begin{array}{l}\text { M. officinalis }(n=6) \\
\text { Placebo }(n=6)\end{array}$ & $\begin{array}{l}922.17 \pm 460.45 \\
924.50 \pm 552.37\end{array}$ & $\begin{array}{l}959.67 \pm 527.59 \\
882.67 \pm 487.78\end{array}$ & $\begin{array}{l}37.50 \pm 141.19 \\
-41.83 \pm 180.65\end{array}$ & 0.367 \\
\hline CSF-ptau concentration & $\begin{array}{l}\text { M. officinalis }(n=7) \\
\text { Placebo }(n=6)\end{array}$ & $\begin{array}{l}106.14 \pm 41.44 \\
91.17 \pm 31.92\end{array}$ & $\begin{array}{l}114.57 \pm 41.60 \\
95.33 \pm 29.81\end{array}$ & $\begin{array}{l}8.43 \pm 17.19 \\
4.17 \pm 6.85\end{array}$ & 0.558 \\
\hline
\end{tabular}

Table 4. Mean and estimated mean changes in disease-related biomarker measures from the baseline to 24-week follow-up. A $\beta$ amyloid $\beta$-protein; CSF cerebrospinal fluid; FDG fluorodeoxyglucose; M. officinalis, Melissa officinalis; PET positron emission tomography; PiB Pittsburgh compound-B; ptau phosphorylated tau 181p; SUVR standardized uptake value ratio; tau total tau protein; VSRAD Voxel-Based Specific Regional Analysis System for Alzheimer's disease.

$53.58 \pm 46.55 \mathrm{nmol} / \mathrm{L}$, respectively (Fig. 3). Conjugated form of RA reached peaked at 16 weeks and slightly decreased at 24 weeks, while intact form of RA gradually increased up to 24 weeks.

RA was not detectable in the serum at the baseline (prior to treatment with $M$. officinalis extract) in the intervention group or at any time point during the trial period in the placebo group, except in the serum collected from one subject in the placebo group; surprisingly, in this subject, total RA in the serum at the baseline, 8-, 16-, and 24 -week visits was $30.63,30.06,13.20$, and $47.22 \mathrm{nmol} / \mathrm{L}$, respectively. Additionally, in one M. officinalis group patient, RA in the serum could be detected only at the 16 -week visit $(32.58 \mathrm{nmol} / \mathrm{L})$ but not at the 8 - and 24 -week visits, as the concentrations were below the level of detection; the study partner of this patient reported adherence as $88.00 \%$. RA levels were undetectable $\left(<0.28 \times 10^{-2} \mathrm{nmol} / \mathrm{L}\right)$ in the CSF of patients in both the placebo and M. officinalis groups at the baseline and the 24-week follow-up. 


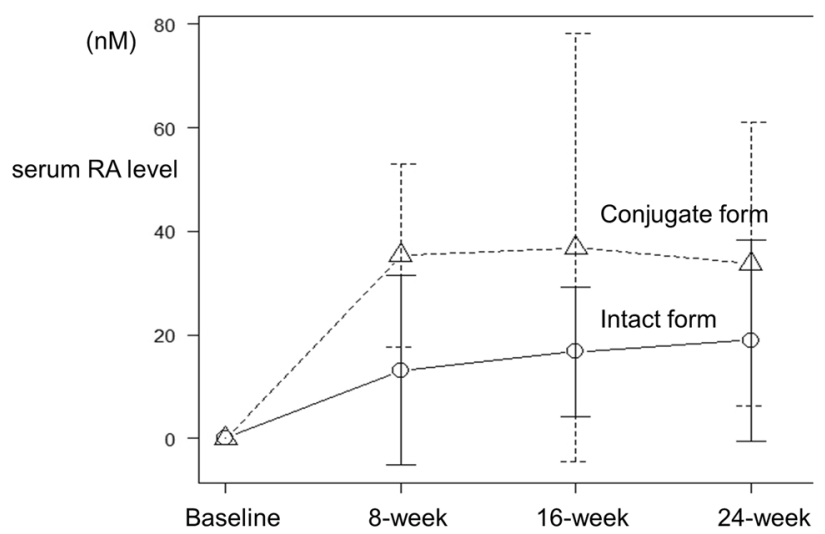

Figure 3. Mean serum RA levels at each study time point in the M. officinalis group. Open triangles represent the conjugated form of rosmarinic acid (RA), and open circles represent the intact form of RA.

\section{Discussion}

In the 24-week double-blind placebo-controlled study, with a 24-week extension, we examined the safety and tolerability of $M$. officinalis extract containing RA ( $500 \mathrm{mg}$ daily dose) in patients with mild dementia due to AD as a primary endpoint and demonstrated that supplementation with this dose was tolerable for 48 weeks and did not raise any safety concerns that manifested clinically or through routine blood tests.

We examined the influence of $M$. officinalis extract containing RA on clinical assessments and biomarkers as secondary endpoints. No differences were detected between the treatment and placebo groups in terms of cognitive performance or daily function between the baseline and 24-week follow-up visits; however, a significant time $\times$ treatment group interaction was observed for the NPI-Q score and its subscale "Irritability/Lability", suggesting benefits of the $M$. officinalis extract in the management of irritability or lability in patients with AD. Consistent with our results, $M$. officinalis extract and aromatherapy with the essential oil of $M$. officinalis have been shown to have a positive effect on agitation in patients with mild to moderate AD and severe dementia, respectively ${ }^{16,18}$. In addition, $M$. officinalis essential oil was reported to decrease NPI scores in patients without dementia, implying that it reduces agitation ${ }^{19}$. M. officinalis has both nicotinic and muscarinic acetylcholine receptor binding activity; however, the specific action of $M$. officinalis on acetylcholine receptors remains unknown ${ }^{20}$, and whether it shows efficacy in the treatment of patients with AD with respect to not only their behavioral and psychological symptoms of dementia, such as irritability, but also their cognition is unclear ${ }^{20}$. Further, we were unable to reveal biological evidence of efficacy against AD pathophysiology about changes in amyloid or tau markers in this trial with about 10 patients in each group. Overall, we were able to provide a little evidence for the clinical efficacy of $M$. officinalis extract as the secondary outcomes of this study.

This is the first randomized double-blind placebo-controlled study conducted to evaluate RA levels in the serum and in CSF in humans. Interestingly, two participants showed unexpected serum RA levels in the placebo and M. officinalis groups. One patient in the placebo group showed sufficient serum RA concentration. Because some Japanese individuals consume Perilla frutescens ${ }^{10}$, which is rich in RA, our data indicate that this patient might have been eating Perilla frutescens. Further trials should exclude subjects who consume any diets rich in RA during the trial. On the contrary, one subject in the M. officinalis group had considerably low or undetectable levels of serum RA; it is possible that this participant may have had poor medication compliance, in contrast to that reported of the patient's study partner. Our previous study suggests that RA is metabolized and degraded by gut microbiota ${ }^{21}$; therefore, the pharmacokinetics of RA may be affected by the individual states of microbiota. Blood and CSF samplings were performed 4 or $5 \mathrm{~h}$ after the intake of $M$. officinalis extract containing RA (500 mg daily dose). In the $M$. officinalis group, at the 24 -week visit, the serum level of RA and its metabolites was $53.58 \pm 46.55 \mathrm{nmol} / \mathrm{L}$, but the CSF level of RA was below detectable levels $\left(<0.28 \times 10^{-2} \mathrm{nmol} / \mathrm{L}\right)$. Time taken to reach the maximum serum concentration of RA in fed state was reported to be $3 \mathrm{~h}$ after the intake of M. officinalis extract containing RA ${ }^{17}$; higher levels of RA in serum and CSF might have been observed if serum and CSF were collected at an earlier timing. Pharmacokinetic study revealed that conjugated form of RA in serum reached peak at 16 weeks and slightly decreased at 24 weeks, while intact form of RA in serum gradually increased up to 24 weeks. This result might suggest that conjugation ability reached maximum. RA was reported to act as a weak or moderate inhibitor of drug-metabolizing enzymes, such as cytochrome P450 monooxygenase (CYP) uridine and diphosphate glucuronosyltransferase ${ }^{22}$. In the present study, we did not observe any apparent interactions between $M$. officinalis extract and concomitant medications. However, as acetylcholinesterase inhibitors are metabolized by CYP3A4 and CYP2D $6^{23}$, we must pay attention to interactions between RA and concomitant medications especially when patients are treated with anti-AD drugs. Further investigations are necessary to clarify interactions between RA and drugs.

We consider that $M$. officinalis extract containing RA exerted its effects in the brain to prevent the worsening of neuropsychiatric symptoms, although concentrations of RA were considerably low in the CSF. Studies using isotope labeled RA is presumed to be useful to clarify the absorption and distribution of RA in blood, CSF and brain following oral administration. However, as far as we investigated, there have been no studies which show 


\section{Melissa officinalis extract group}

Placebo group

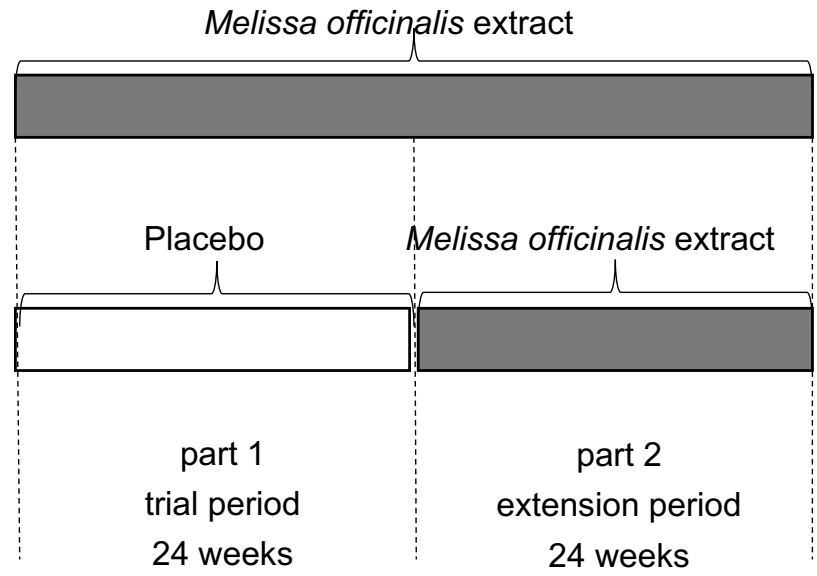

Figure 4. Trial time course. Part 1 of the trial was designed as a randomized double-blind placebo-controlled parallel-group 24-week trial. Patients were randomly allocated in a 1:1 ratio to either the Melissa officinalis group [receiving M. officinalis extract containing $500 \mathrm{mg}$ of rosmarinic acid (RA) daily] or the placebo group. In part 2 (duration of 24 weeks), all patients were assigned to the M. officinalis extract group.

that isotope-labeled RA appears in the central nervous system following oral administration. A previous study with rats demonstrated that intact RA is present in the plasma and brain at mean concentrations of 1440 and $24.1 \mu \mathrm{M}$, respectively (plasma:brain $=60: 1$ ), at $30 \mathrm{~min}$ after the intraperitoneal administration of Plectranthus barbatus extract (containing $\left.250 \mu \mathrm{M} \mathrm{RA} \mathrm{kg}{ }^{-1}\right)^{24}$. It was reported that the ratio of the mean of CSF concentration $\left(\mathrm{C}_{\mathrm{CSF}}\right)$ to that of brain interstitial fluid concentration $\left(\mathrm{C}_{\mathrm{ISF}}\right)\left(\mathrm{C}_{\mathrm{CSF}} / \mathrm{C}_{\mathrm{ISF}}\right)$ for propranolol was 0.49 in nonhuman primates, indicating that $\mathrm{C}_{\mathrm{ISF}}$ was greater than $\mathrm{C}_{\mathrm{CSF}}{ }^{25}$. Additionally, concentration of drug in the brain to $\mathrm{C}_{\mathrm{CSF}}$ ratios was reported to be compound-dependent in rats ${ }^{26}$. Although we did not determine either the $\mathrm{C}_{\mathrm{ISF}}$ of RA or the RA concentrations in the brain in this study, it is possible that the $\mathrm{C}_{\mathrm{ISF}}$ of RA and the RA concentrations of brain might be higher than that of $\mathrm{C}_{\mathrm{CSF}}$

RA might act to suppress AD development even at considerably low concentration in the brain. In our in vivo study of an AD mouse model, RA inhibited A $\beta$ oligomerization and deposition ${ }^{8}$. In addition, we recently reported RA-triggered monoamine increase in the brain, and inhibition of $A \beta$ aggregation by monoamines in mice fed with $\mathrm{RA}^{9}$; however, RA concentrations in the brain of these mice ${ }^{8,9}$ were undetectable. Another possibility is that RA might act on peripheral targets such as gastrointestinal tract, which might contribute to prevention of the worsening of neuropsychiatric symptoms ${ }^{27}$.

The small sample size in this study warrants caution in the interpretation of results and limits their generalization. RA has been reported to decrease $A \beta$ oligomer-induced synaptic toxicities ${ }^{5}$. Because pathogenic $A \beta$ oligomers appear early in the disease ${ }^{28}$, treatment of $\mathrm{AD}$ using RA should be employed earlier in the disease process; for example, at the preclinical stage. A community-based intervention trial using $M$. officinalis extract containing RA aimed at the prevention of dementia is currently ongoing ${ }^{29}$.

This randomized double-blind placebo-controlled study on patients with mild AD dementia demonstrated that M. officinalis extract containing RA (500 mg daily dose) was safe and well-tolerated and that RA may provide a little help to control the worsening of neuropsychiatric symptoms in mild AD dementia.

\section{Materials and methods}

Patient population. Patients with mild dementia due to $\mathrm{AD}$ were enrolled at the Kanazawa University Hospital. The inclusion criteria were as follows: (i) diagnosis of probable AD dementia with high evidence of the pathophysiological process, according to the criteria of the National Institute on Aging-Alzheimer's Association Workgroup (NIA-AA) ${ }^{30}$; (ii) age greater than 59 years; (iii) MMSE scores ${ }^{31}$ between 20 and 26; (iv) CDR score ${ }^{32}$ of 0.5 or 1 (the CDR score ranges from 0 to 3, with higher scores indicating worsening dementia); (v) availability of a study partner to monitor the administration of medication; (vi) stable dosage of acetylcholinesterase inhibitors and memantine for 60 days before screening. The exclusion criteria were as follows: (i) a significant systemic illness such as liver, renal, or heart dysfunction, (ii) a history of cancer, (iii) allergies to polyphenols or any drug or food ingredient, and (iv) subjects who consume any supplements containing RA. Additionally, magnetic resonance imaging (MRI), ${ }^{11} \mathrm{C}-\mathrm{PiB} \mathrm{PET}$, and ${ }^{18} \mathrm{~F}-\mathrm{FDG}$ PET were performed to exclude patients with alternative causes of dementia. Furthermore, the number of apolipoprotein E (APOE) E4 carriers were evaluated in the placebo and M. officinalis groups. The APOE phenotype was determined using isoelectric electrophoresis, as described by Kamboh et al. ${ }^{33}$.

This study was conducted according to the guidelines of the Declaration of Helsinki, and all procedures involving human participants were approved by the Kanazawa University Medical Ethics Review Board (approval number 932). The study was registered at the University Hospital Medical Information Network Clinical Trials Registry (UMIN-CTR: UMIN000007734) (16/04/2012). Written informed consent was obtained from all patients or their legal representatives. An independent data monitoring committee (IDMC) monitored the progress of the trial. 
Intervention. This study consisted of a randomized double-blind placebo-controlled parallel-group 24-week trial period (part 1), followed by an extension period of 24 weeks (part 2). In part 2, all patients were allocated to the M. officinalis group. Figure 4 illustrates the time course of the trial.

Study visits were set up at screening, baseline, and 8-, 16-, 24-, 32-, 40-, and 48-week follow-ups. Randomization was stratified according to age and gender. All patients were randomly allocated in a 1:1 ratio to receive the intervention (M. officinalis extract) or placebo. Block randomization was used to maintain a balance of three age-strata $(60-69,70-79$, and $>80$ years of age $)$ and gender.

Primary outcome measures. The primary outcome was the safety and tolerability of M. officinalis extract containing RA on ingestion. Safety assessments included the vital sign evaluation, physical and neurological examinations, adverse event evaluation, urinalysis, and routine laboratory tests, including hematology (white blood cell count, red blood cell count, hemoglobin level, hematocrit, and platelet count) and blood biochemistry analysis (levels of AST, ALT, LDH, T-bil, BUN, and Cr), electrocardiography, and chest X-rays. Blood samples were collected before lunch. Most of the patients ingested the placebo or M. officinalis extract capsules 4 or $5 \mathrm{~h}$ before blood sample collection. CSF was collected at the same time as blood samples. All safety information was reviewed by the IDMC at quarterly intervals. Unused $M$. officinalis extract or placebo capsules were stored by the patients, brought back to the investigating physician during each follow-up consultation, and counted to measure adherence.

Secondary outcome measures. The secondary outcomes included changes in clinical assessments and biomarkers. An array of cognitive and neuropsychiatric measures was administered at baseline and during the 8-, 16-, and 24-week follow-up assessments. The measures included an ADAS-cog (score range $=0-70$, with higher scores indicating worsening dementia $)^{34}$, MMSE, assessment of dementia according to the CDR, assessment of daily function according to the DAD (score range $=0-40$, with lower scores indicating worsening dementia) ${ }^{35}$, and NPI-Q (score range $=0-36$, with higher scores indicating more severe symptoms) ${ }^{36}$. Biomarkers included ${ }^{11} \mathrm{C}-\mathrm{PiB}$ PET, ${ }^{18} \mathrm{~F}-\mathrm{FDG}$ PET, volumetric MRI, and CSF biochemical markers [A $\beta_{1-42}$, tau, and ptau]. The change from the baseline to the 24 -week follow-up visit was assessed based on the ${ }^{11} \mathrm{C}-\mathrm{PiB}$ SUVR ${ }^{37,38},{ }^{18} \mathrm{~F}$-FDG PET $\mathrm{z}$-scores (the degree of regional metabolic reduction) ${ }^{39,40}$, MRI z-scores (the degree of atrophy in the entorhinal cortex $^{41}$, and CSF markers ${ }^{42,43}$. Details of methods for PET, MRI and CSF marker studies are summarized in Additional Method 1.

Exploratory examination. Concentrations of intact and conjugated RA were assessed in the serum at baseline and during the 8-, 16-, and 24-week follow-up visits, and in CSF at baseline and the 24-week follow-up. RA levels in the serum were measured using high-performance liquid chromatography (HPLC), as described previously $\mathrm{y}^{44,45}$, with the lower limit of detection as $10 \mathrm{nmol} / \mathrm{L}$. RA levels in the CSF were measured using liquid chromatography coupled with electrospray ionization tandem mass spectrometry (LC-ESI-MS/MS). The limit of detection for intact and conjugated RA in the CSF samples was $0.28 \times 10^{-2} \mathrm{nmol} / \mathrm{L}$. Details of quantification of RA levels in the serum and CSF are summarized in Additional Method 2.

Statistical analyses. The sample size was determined based on the primary objective, i.e., to assess the safety and tolerability of $M$. officinalis extract containing RA.

Measurements used to determine the safety of $M$. officinalis extract containing RA were analyzed using all the available data from all available subjects (full-analysis set). Analysis of the efficacy of M. officinalis extract containing RA was performed using data collected from patients who completed the whole study because of the lack of follow-up biomarker data for subjects who discontinued the project (per protocol set) in addition to full-analysis set. Generalized linear mixed effects repeated measures analysis was used to analyze the effect of treatment on changes in all measures from baseline to the 8-, 16-, 24-, 32-, 40-, and 48-week follow-up visits. Data were reported as mean \pm standard deviation, unless specified otherwise. A $P$ value of $<0.05$ was considered statistically significant. All statistical analyses were performed using the SPSS software (version 23; SPSS Inc., Chicago, IL, USA).

\section{Data availability}

The datasets used and analyzed during the current study are available from the corresponding author on reasonable request.

\section{Code availability}

The full trial protocol can be accessed in https://upload.umin.ac.jp/cgi-open-bin/ctr/ctr_view.cgi?recptno=R0000 09110 (English and Japanese).

Received: 26 June 2020; Accepted: 22 September 2020

Published online: 29 October 2020

\section{References}

1. Hardy, J. \& Selkoe, D. J. The amyloid hypothesis of Alzheimer's disease: progress and problems on the road to therapeutics. Science 297, 353-356. https://doi.org/10.1126/science.1072994 (2002).

2. Jack, C. R. Jr. et al. Tracking pathophysiological processes in Alzheimer's disease: an updated hypothetical model of dynamic biomarkers. Lancet Neurol. 12, 207-216. https://doi.org/10.1016/S1474-4422(12)70291-0 (2013). 
3. Petersen, M. \& Simmonds, M. S. Rosmarinic acid. Phytochemistry 62, 121-125. https://doi.org/10.1016/s0031-9422(02)00513-7 (2003).

4. Ono, K., Hasegawa, K., Naiki, H. \& Yamada, M. Curcumin has potent anti-amyloidogenic effects for Alzheimer's beta-amyloid fibrils in vitro. J. Neurosci. Res. 75, 742-750. https://doi.org/10.1002/jnr.20025 (2004).

5. Ono, K. et al. Phenolic compounds prevent amyloid beta-protein oligomerization and synaptic dysfunction by site-specific binding. J. Biol. Chem. 287, 14631-14643. https://doi.org/10.1074/jbc.M111.325456 (2012).

6. Martin, S. J., Grimwood, P. D. \& Morris, R. G. Synaptic plasticity and memory: an evaluation of the hypothesis. Annu. Rev. Neurosci. 23, 649-711. https://doi.org/10.1146/annurev.neuro.23.1.649 (2000).

7. Alkam, T., Nitta, A., Mizoguchi, H., Itoh, A. \& Nabeshima, T. A natural scavenger of peroxynitrites, rosmarinic acid, protects against impairment of memory induced by Abeta(25-35). Behav. Brain Res. 180, 139-145. https://doi.org/10.1016/j.bbr.2007.03.001 (2007).

8. Hamaguchi, T., Ono, K., Murase, A. \& Yamada, M. Phenolic compounds prevent Alzheimer's pathology through different effects on the amyloid-beta aggregation pathway. Am. J. Pathol. 175, 2557-2565. https://doi.org/10.2353/ajpath.2009.090417 (2009).

9. Hase, T. et al. Rosmarinic acid suppresses Alzheimer's disease development by reducing amyloid beta aggregation by increasing monoamine secretion. Sci. Rep. 9, 8711. https://doi.org/10.1038/s41598-019-45168-1 (2019).

10. Makino, T., Ono, T., Muso, E. \& Honda, G. Inhibitory effect of Perilla frutescens and its phenolic constituents on cultured murine mesangial cell proliferation. Planta Med. 64, 541-545. https://doi.org/10.1055/s-2006-957510 (1998).

11. Al-Sereiti, M. R., Abu-Amer, K. M. \& Sen, P. Pharmacology of rosemary (Rosmarinus officinalis Linn.) and its therapeutic potentials. Indian J. Exp. Biol. 37, 124-130 (1999).

12. Areias, F., Valentao, P., Andrade, P. B., Ferreres, F. \& Seabra, R. M. Flavonoids and phenolic acids of sage: influence of some agricultural factors. J. Agric. Food Chem. 48, 6081-6084. https://doi.org/10.1021/jf000440+ (2000).

13. Carnat, A. P., Carnat, A., Fraisse, D. \& Lamaison, J. L. The arimatic and polyphenolic composition of lemon balm (Mellisa officinalis L. subsup. officinalis) tea. Pharm. Acta Helv. 72, 301-305 (1998).

14. Kennedy, D. O., Scholey, A. B., Tildesley, N. T., Perry, E. K. \& Wesnes, K. A. Modulation of mood and cognitive performance following acute administration of Melissa officinalis (lemon balm). Pharmacol. Biochem. Behav. 72, 953-964. https://doi.org/10.1016/ s0091-3057(02)00777-3 (2002).

15. Kennedy, D. O. et al. Modulation of mood and cognitive performance following acute administration of single doses of Melissa officinalis (Lemon balm) with human CNS nicotinic and muscarinic receptor-binding properties. Neuropsychopharmacology 28, 1871-1881. https://doi.org/10.1038/sj.npp.1300230 (2003).

16. Akhondzadeh, S. et al. Melissa officinalis extract in the treatment of patients with mild to moderate Alzheimer's disease: a double blind, randomised, placebo controlled trial. J. Neurol. Neurosurg. Psychiatry 74, 863-866. https://doi.org/10.1136/jnnp.74.7.863 (2003).

17. Noguchi-Shinohara, M. et al. Pharmacokinetics, safety and tolerability of Melissa Officinalis extract which contained rosmarinic acid in healthy individuals: a randomized controlled trial. PLOS ONE 10, e0126422. https://doi.org/10.1371/journal.pone.01264 22 (2015).

18. Ballard, C. G., O'Brien, J. T., Reichelt, K. \& Perry, E. K. Aromatherapy as a safe and effective treatment for the management of agitation in severe dementia: the results of a double-blind, placebo-controlled trial with Melissa. J. Clin. Psychiatry 63, 553-558. https://doi.org/10.4088/jcp.v63n0703 (2002).

19. Watson, K., Hatcher, D. \& Good, A. A randomised controlled trial of Lavender (Lavandula Angustifolia) and Lemon Balm (Melissa Officinalis) essential oils for the treatment of agitated behaviour in older people with and without dementia. Complement. Ther. Med. 42, 366-373. https://doi.org/10.1016/j.ctim.2018.12.016 (2019).

20. Wake, G. et al. CNS acetylcholine receptor activity in European medicinal plants traditionally used to improve failing memory. J. Ethnopharmacol. 69, 105-114. https://doi.org/10.1016/s0378-8741(99)00113-0 (2000).

21. Konishi, Y. \& Kobayashi, S. Transepithelial transport of rosmarinic acid in intestinal Caco-2 cell monolayers. Biosci. Biotechnol. Biochem. 69, 583-591. https://doi.org/10.1271/bbb.69.583 (2005).

22. Kim, S. B., Kim, K. S., Kim, D. D. \& Yoon, I. S. Metabolic interactions of rosmarinic acid with human cytochrome P450 monooxygenases and uridine disphosphate glucuronosyltransferases. Biomed. Pharmacother. 110, 111-117. https://doi.org/10.1016/j.bioph a.2018.11.040 (2019).

23. Cummings, J. L. Alzheimer's disease. N. Engl. J. Med. 351, 56-67. https://doi.org/10.1056/NEJMra040223 (2004).

24. Fale, P. L., Madeira, P. J., Florencio, M. H., Ascensao, L. \& Serralheiro, M. L. Function of Plectranthus barbatus herbal tea as neuronal acetylcholinesterase inhibitor. Food Funct. 2, 130-136. https://doi.org/10.1039/c0fo00070a (2011).

25. Nagaya, Y. et al. Utility of cerebrospinal fluid drug concentration as a surrogate for unbound brain concentration in nonhuman primates. Drug Metab. Pharmacokinet. 29, 419-426. https://doi.org/10.2133/dmpk.dmpk-14-rg-026 (2014).

26. Huang, L., Li, X., Roberts, J., Janosky, B. \& Lin, M. H. Differential role of P-glycoprotein and breast cancer resistance protein in drug distribution into brain, CSF and peripheral nerve tissues in rats. Xenobiotica 45, 547-555. https://doi.org/10.3109/00498 254.2014.997324 (2015).

27. Mörkl, S., Butler, M. I., Cryan, J. F. \& Dinan, T. G. Probiotics and the microbiota-gut-brain axis: focus on psychiatry. Curr. Nutr. Rep. 9, 171-182. https://doi.org/10.1007/s13668-020-00313-5 (2020).

28. Viola, K. L. \& Klein, W. L. Amyloid beta oligomers in Alzheimer's disease pathogenesis, treatment, and diagnosis. Acta Neuropathol. 129, 183-206. https://doi.org/10.1007/s00401-015-1386-3 (2015).

29. NIPH Clinical trials registry. In:https://jrct.niph.go.jp/latest-detail/jRCTs041180064. Accessed 25 June 2020.

30. McKhann, G. M. et al. The diagnosis of dementia due to Alzheimer's disease: recommendations from the National Institute on Aging-Alzheimer's Association workgroups on diagnostic guidelines for Alzheimer's disease. Alzheimers Dement 7, 263-269. https ://doi.org/10.1016/j.jalz.2011.03.005 (2011).

31. Folstein, M. F., Folstein, S. E. \& McHugh, P. R. "Mini-mental state". A practical method for grading the cognitive state of patients for the clinician. J. Psychiatr. Res. 12, 189-198. https://doi.org/10.1016/0022-3956(75)90026-6 (1975).

32. Morris, J. C. The Clinical Dementia Rating (CDR): current version and scoring rules. Neurology 43, 2412-2414. https://doi. org/10.1212/wnl.43.11.2412-a (1993).

33. Kamboh, M. I., Ferrel, R. E. \& Kottke, B. Genetic studies of human apolipoproteins V: a novel rapid procedure to screen apolipoprotein E polymorohism. J. Lipid Res. 29, 1535-1543 (1998).

34. Rosen, W. G., Mohs, R. C. \& Davis, K. L. A new rating scale for Alzheimer's disease. Am. J. Psychiatry 141, 1356-1364. https://doi. org/10.1176/ajp.141.11.1356 (1984).

35. Gelinas, I., Gauthier, L., McIntyre, M. \& Gauthier, S. Development of a functional measure for persons with Alzheimer's disease: the disability assessment for dementia. Am. J. Occup. Ther. 53, 471-481. https://doi.org/10.5014/ajot.53.5.471 (1999).

36. Kaufer, D. I. et al. Validation of the NPI-Q, a brief clinical form of the Neuropsychiatric Inventory. J. Neuropsychiatry Clin. Neurosci. 12, 233-239. https://doi.org/10.1176/jnp.12.2.233 (2000).

37. Tzourio-Mazoyer, N. et al. Automated anatomical labeling of activations in SPM using a macroscopic anatomical parcellation of the MNI MRI single-subject brain. Neuroimage 15, 273-289. https://doi.org/10.1006/nimg.2001.0978 (2002).

38. Mormino, E. C. et al. Episodic memory loss is related to hippocampal-mediated beta-amyloid deposition in elderly subjects. Brain 132, 1310-1323. https://doi.org/10.1093/brain/awn320 (2009). 
39. Matsunari, I. et al. Comparison of 18F-FDG PET and optimized voxel-based morphometry for detection of Alzheimer's disease: aging effect on diagnostic performance. J. Nucl. Med. 48, 1961-1970. https://doi.org/10.2967/jnumed.107.042820 (2007).

40. Matsuda, H. Role of neuroimaging in Alzheimer's disease, with emphasis on brain perfusion SPECT. J. Nucl. Med. 48, 1289-1300. https://doi.org/10.2967/jnumed.106.037218 (2007).

41. Hirata, Y. et al. Voxel-based morphometry to discriminate early Alzheimer's disease from controls. Neurosci. Lett. 382, $269-274$. https://doi.org/10.1016/j.neulet.2005.03.038 (2005).

42. Maddalena, A. et al. Biochemical diagnosis of Alzheimer disease by measuring the cerebrospinal fluid ratio of phosphorylated tau protein to beta-amyloid peptide42. Arch. Neurol. 60, 1202-1206. https://doi.org/10.1001/archneur.60.9.1202 (2003).

43. Hulstaert, F. et al. Improved discrimination of AD patients using beta-amyloid(1-42) and tau levels in CSF. Neurology 52, 15551562. https://doi.org/10.1212/wnl.52.8.1555 (1999).

44. Konishi, Y., Hitomi, Y., Yoshida, M. \& Yoshioka, E. Pharmacokinetic study of caffeic and rosmarinic acids in rats after oral administration. J. Agric. Food Chem. 53, 4740-4746. https://doi.org/10.1021/jf0478307 (2005).

45. Baba, S. et al. Absorption, metabolism, degradation and urinary excretion of rosmarinic acid after intake of Perilla frutescens extract in humans. Eur. J. Nutr. 44, 1-9. https://doi.org/10.1007/s00394-004-0482-2 (2005).

\title{
Acknowledgements
}

The authors thank the participants and their study partners. The authors also thank Ms. Chiaki Dohmoto for psychological evaluation. The authors thank Enago (www.enago.jp) for the English language review.

\section{Author contributions}

M.N-S. was co-principal investigator on the study, collaborated on the original proposal, drafted the manuscript and contributed the acquisition, analysis, and interpretation of data. K.O. was co-principal investigator on the study, collaborated on the original proposal, drafted the manuscript and contributed the acquisition of data. T.H. was co-principal investigator on the study, collaborated on the original proposal. T.N. and S.K. measured rosmarinic acid level in serum and CSF, collaborated on the original proposal and reviewed the manuscript. J.K. and M.S-Y. were co-principal investigator on the study, supervised section of the PET tests, contributed the analysis of data regarding PET tests and reviewed the manuscript. K.I. was co-principal investigator on the study, collaborated on the original proposal. K.Y. was co-principal investigator on the study, supervised section of the PET tests. H.N. was an expert of statistics, advised the statistical analysis. M.Y. was the principal investigator on the study, designed the study and directed its implementation, including quality assurance and control.

\section{Funding}

This trial is supported in part by a Grant-in-Aid Scientific Research from the Science of the Ministry of Education, Culture, Sports, Science and Technology of Japan (M. N-S, Grant Number 23790984), by a grant for the Amyloidosis Research Committee from the Ministry of Health, Labour and Welfare, Japan (M.Y, Grant Number H17-Nanchi-Ippan 012, H20-Nanchi-Ippan 012). This research was supported by AMED under Grant Number JP15dk0207016h0002 (M.N-S), JP18dk0207021 (S.K), and by Council for Science, Technology and Innovation (CSTI), Cross-ministerial Strategic Innovation Promotion Program (SIP) (14532924 to M.Y and S.K). The sponsors had no role in the design and conduct of the study; collection, management, analysis, and interpretation of the data; or preparation, review, or approval of the manuscript.

\section{Competing interests}

The authors declare no competing interests.

\section{Additional information}

Supplementary information is available for this paper at https://doi.org/10.1038/s41598-020-73729-2.

Correspondence and requests for materials should be addressed to M.Y.

Reprints and permissions information is available at www.nature.com/reprints.

Publisher's note Springer Nature remains neutral with regard to jurisdictional claims in published maps and institutional affiliations.

\begin{abstract}
Open Access This article is licensed under a Creative Commons Attribution 4.0 International License, which permits use, sharing, adaptation, distribution and reproduction in any medium or format, as long as you give appropriate credit to the original author(s) and the source, provide a link to the Creative Commons licence, and indicate if changes were made. The images or other third party material in this article are included in the article's Creative Commons licence, unless indicated otherwise in a credit line to the material. If material is not included in the article's Creative Commons licence and your intended use is not permitted by statutory regulation or exceeds the permitted use, you will need to obtain permission directly from the copyright holder. To view a copy of this licence, visit http://creativecommons.org/licenses/by/4.0/.
\end{abstract}

(C) The Author(s) 2020 